\title{
Quasiperiodic waves at the onset of zero Prandtl number convection with rotation
}

\author{
Krishna Kumar ${ }^{1}$, Sanjay Chaudhuri ${ }^{2}$, and Alaka Das ${ }^{1}$ \\ ${ }^{1}$ Physics and Applied Mathematics Unit \\ ${ }^{2}$ Statistics and Mathematics Unit \\ Indian Statistical Institute \\ 203, Barrackpore Trunk Road, Calcutta-700 035, India
}

12 April, 2001

\begin{abstract}
We show the possibility of quasiperiodic waves at the onset of thermal convection in a thin horizontal layer of slowly rotating zeroPrandtl number Boussinesq fluid confined between stress-free conducting boundaries. Two independent frequencies emerge due to an interaction between a stationary instability and a self-tuned wavy instability in presence of coriolis force, if Taylor number is raised above a critical value. Constructing a dynamical system for the hydrodynamical problem, the competition between the interacting instabilities is analyzed. The forward bifurcation from the conductive state is selftuned.
\end{abstract}

PACS number(s):47.20.Ky, 47.27.Te 
Thermal convection in Boussinesq fluids in the limit of vanishing Prandtl number $P(=\nu / \kappa)$ ([1]-[12]) is of interest for astrophysical problems $(P \approx$ $\left.10^{-8}\right)$ as well as for liquid metals $\left(P \approx 10^{-2}-10^{-3}\right)$. The theoretical study, in particular with stress-free boundary conditions, in the limit of large thermal diffusitivity $\kappa$ has been considered subtle for a long time because the linearly unstable two-dimensional (2D) rolls become exact nonlinear solution. The nonlinearity $\mathbf{v} \cdot \nabla \theta$ due to the advection of temperature fluctuation $\theta$ by the velocity field $\mathbf{v}$ might be negligible [1]. The nonlinearity $\mathbf{v} \cdot \nabla \mathbf{v}$, due to the self interaction of velocity field, does not contribute to saturation for straight (2D) rolls. This led to the speculation that the zero $P$ limit might involve a singular limit problem similar to the one with infinite Reynold number in incompressible fluid dynamics. However, the recent three-dimensional (3D) direct numerical simulation (DNS) of zero-Prandtl number Boussinesq equations by Thual [11] showed the saturation instead of indefinite growth of the solution even with stress-free boundary conditions. He also compared the results of zero P equations with that of the full Oberbeck-Boussinesq equations in the asymptotic limit of vanishing $\mathrm{P}$, and found complete agreement in two cases. The saturation of growing $2 \mathrm{D}$ rolls at the onset of convection occurs by generation of self-tuned 3D waves, the mechanism of which was explained in a simple model [12]. The results of this model, in its validity range, agreed well with that of DNS just above the convective instability. The new nonlocal instability at the onset occurs purely due to nonlinear effects, while linear equations predict the stationary instability [13].

We present, in this article, a dynamical system constructed for thermal convection in zero Prandtl number Boussinesq fluid, confined between stressfree conducting flat boundaries, and subjected to a slow rotation about the vertical axis. We then investigate numerically the system to study the effect of coriolis force on the onset of convection. We show that the convection sets in as quasiperiodic waves at the onset of convective instability for Taylor number $T$ above a critical value $T_{c}$, although the principle of exchange of stability is valid according to the linear theory [13] for these Taylor numbers. The generation of two independent frequencies is the result of an interaction between a stationary instability and a self-tuned wavy perturbations in presence of coriolis force. This an example of a new self-tuned forward bifurcation. For the values of Taylor number below $T_{c}$, the convection sets in as self-tuned wavy instability as is the case in absence of rotation. However, the model shows a possibility transition from one wavy instability to another through a narrow window of period-doubling instability. 
We consider a thin layer of a Boussinesq fluid of infinite horizontal extension subjected to a uniform adverse temperature gradient $\beta$ across the fluid layer, and a rigid body rotation with an angular velocity $\Omega$ about the vertical axis. The fluid is assumed to have uniform values of the kinematic viscosity $\nu$ and the thermal difusitivity $\kappa$. The basic state is the conductive state with no fluid motion in the rotating frame of reference. The convective flow, in the limit of zero-Prandtl number, is then described by the following system of dimesionless hydrodynamic equations,

$$
\begin{aligned}
\partial_{t}\left(\nabla^{2} v_{3}\right) & =\nabla^{4} v_{3}+R \nabla_{H}^{2} \theta-\sqrt{T} \partial_{z} \omega_{3} \\
& -\hat{e}_{3} \cdot \nabla \times[(\omega \cdot \nabla) \mathbf{v}-(\mathbf{v} \cdot \nabla) \omega] \\
\partial_{t} \omega_{3} & =\nabla^{2} \omega_{3}+\sqrt{T} \partial_{z} v_{3} \\
& +\left[(\omega \cdot \nabla) v_{3}-(\mathbf{v} \cdot \nabla) \omega_{3}\right], \\
\nabla^{2} \theta & =-v_{3},
\end{aligned}
$$

where $\mathbf{v}(x, y, z, t) \equiv\left(v_{1}, v_{2}, v_{3}\right)$ is the velocity field, $\theta(x, y, z, t)$ the deviation in temperature field from steady conduction profile, $\omega \equiv\left(\omega_{1}, \omega_{2}, \omega_{3}\right)=\nabla \times \mathbf{v}$ the vorticity field of the fluid. In the above, length scales are made dimensionless by the thickness $d$ of the fluid layer, time by the viscous time scale $\frac{d^{2}}{\nu}$, and the temperature field by $(\beta d) \frac{\nu}{\kappa}$. Rayleigh number $R=\frac{\alpha g \beta d^{4}}{\nu \kappa}$ and Taylor number $T=\frac{4 d^{4} \Omega^{2}}{\nu^{2}}$ are the two dimensionless external parameters. The unit vector $\hat{e}_{3}$ is directed vertically upward. We impose periodic boundary conditions in horizontal plane. This introduces two fundamental wave numbers $k$ along $\mathrm{x}$-axis and $q$ along y-axis. The stress-free boundary conditions imply $\partial_{z} v_{1}=\partial_{z} v_{2}=v_{3}=0$ at $z=0,1$. Thermally conducting horizontal boundaries mean $\theta=0$ at $z=0,1$.

The hydrodynamical equations (1-3) are the same as those derived by Chandrasekhar [13. We have nondimesionlized them and considered the case of zero $P$. We have also eleminited the pressure term from NavierStokes equations by taking curl twice and using the incompressibility condition $(\nabla \cdot \mathbf{v}=0)$. The conclusions derived from the linearized version of the equations remain unchanged even in the present case. Following the arguments of Chandrasekhar [13], one arrives at the conclusion that the principle of exchange of stability is valid even in the limit discussed here. The critical value of Rayleigh number now reads $R_{c}(T)=\frac{\pi^{4}}{x}\left[(1+x)^{3}+\frac{T}{\pi^{4}}\right]$ and critical wave number $k_{c}(T)=\pi \sqrt{\left(l_{1}+l_{2}-\frac{1}{2}\right)}$ now depend on Taylor number 
$T$ [13]. In the above, $l_{1,2}=\left[\frac{1}{4}\left\{\frac{1}{2}+\frac{T}{\pi^{4}} \pm \sqrt{\left(\frac{1}{2}+\frac{T}{\pi^{4}}\right)-\frac{1}{4}}\right\}\right]^{\frac{1}{3}}$ and $x=k_{c}^{2} / \pi^{2}$. In absence of rotation $k_{c}^{0} \equiv k_{c}(T=0)=\pi / \sqrt{2}$ and $R_{c}^{0} \equiv R_{c}(T=0)=$ $27 \pi^{4} / 4$.

The 2D rolls are not exact solutions of nonlinear hydrodynamic system with rotation as is the case in zero-Prandtl number convection in absence of rotation. Nevertheless, the growing 2D rolls are not saturated just above the onset of convective instability. The saturation occurs only because of nonlinear interaction of $2 \mathrm{D}$ rolls with $3 \mathrm{D}$ wavy perturbations. To understand the nonlinear behavior close to the convective instability, we construct a consistent minimal-mode model using Galerkin technique [14]. We expand the vertical velocity $v_{3}$ and the vertical vorticity $\omega_{3}$ in Fourier series compatible with the stress-free boundary conditions and conducting thermal boundary conditions. As the DNS, in absence of rotation, showed standing patterns 12] instead of traveling patterns, we expect similar behavior at least for small rotation rates. Therefore, we expand the fields with real Fourier coefficients. This lead to the following expansion for the vertical velocity and the vertical vorticity in a minimum-mode model.

$$
\begin{aligned}
v_{3}(x, y, z, t) & =W_{101}(t) \cos k_{c} x \sin \pi z \\
& +W_{111}(t) \cos k_{c} x \cos q y \sin \pi z \\
& +W_{\overline{1} \overline{1} 1}(t) \sin k_{c} x \sin q y \sin \pi z \\
& +W_{012}(t) \cos q y \sin 2 \pi z+\ldots \\
\omega_{3}(x, y, z, t) & =\zeta_{101}(t) \cos k_{c} x \cos \pi z \\
& +\zeta_{010}(t) \cos q y+\zeta_{\overline{1} \overline{1} 0}(t) \sin k_{c} x \sin q y \\
& +\zeta_{111}(t) \cos k_{c} x \cos q y \cos \pi z \\
& +\zeta_{012}(t) \cos q y \cos 2 \pi z+\zeta_{200}(t) \cos 2 k_{c} x \\
& +\zeta_{210}(t) \cos 2 k_{c} x \cos q y \\
& +\zeta_{\overline{2} \overline{1} 0}(t) \sin 2 k_{c} x \sin q y+\ldots
\end{aligned}
$$

The mode selection is quite systematic. As rotation couples the vertical velocity and the vertical vorticity linearly, we have selected the mode $\zeta_{101}$. The mode $\zeta_{010}$ is essential to saturate zero Prandtl number convection via wavy instability. All other modes appear through the nonlinear interaction of these vorticity modes with the critical velocity mode $W_{101}$. As the vorticity field is very crucial for saturation in the limit of vanishing Prandtl number, all relevant second harmonics are retained for vertical vorticity. All relevant 
harmonics of the vertical velocity field, consistent with the selection of the vertical vorticity, are also retained. Other higher order modes may be required as Rayleigh number is raised further. As we are interested to capture essential nonlinear interaction between competing instabilities just above the onset of convection, these modes are essential. The solenoidal character of the velocity and the vorticity fields yield horizontal components of the velocity and the vorticity fields. The thermal fluctuation $\theta$ is captured from Eq. 3. Projecting the hydrodynamic equations (11-3) on above modes, we arrive at a twelve-dimensional dynamical system [15].

We now investigate the solutions of the dynamical system by performing numerical integration of the model using standard fourth order Runge-Kutta as well as Bulirsh-Stoer schemes. By choosing a value for $T$, we set $k_{c}(T)$. We then choose a value for $q$. We have tried with different values of the wavenumber $q$ of the perturbations and got qualitatively similar results except when the ratio $q / k_{c}$ is close to unity. We present here all the results for the case $q / k_{c}(T)=0.4$. Initial values for all the twelve modes are chosen randomly, and integration is done for a fixed value of Rayleigh number $R$. We then repeat the process by increasing the value of $R$ in small steps. We have also tried various initial conditions. The results of all the numerical integrations remain the same for the same values of all the relevant parameters. In absence of rotation $(T=0)$, only six modes are excited. This model then reproduces the results of the model [12] of zero $\mathrm{P}$ convection without rotation. In presence of rotation all twelve modes are excited as it should in a consistent model.

Figure 1 gives the stability boundaries of various possible solutions, in the parameter space $R-T$, computed from the model dynamical system. The lowest line in this figure shows linear dependence 13 of $R_{c}$, the critical Rayleigh number, on Taylor number $T$ for the onset of stationary convection in zero Prandtl number Boussinesq fluid. The onset of overstability for the case of vanishing $\mathrm{P}$ for the Taylor numbers considered here is much above, and is not shown in the figure.

As Rayleigh number is raised above its critical value $R_{c}(T)$ for various values of Taylor number below $T=6.0$, conduction state becomes unstable via stationary bifurcation. However, 2D rolls with broken mirror symmetry [16] does not saturate until wavy perturbations interact with them. This saturates the growing rolls at finite amplitude even at the onset. The wavy perturbations are automatically generated when the amplitude of the $2 \mathrm{D}$ roll mode becomes large enough. The self-tuned waves consume the energy of 
$2 \mathrm{D}$ rolls and stop the unbounded growth of latter. This is precisely what happens when there is no rotation and 2D rolls have mirror symmetry.

The three rows of Fig. 2 show projections of the phase diagram, starting from left, in $\zeta_{101}-W_{101}, \zeta_{010}-W_{101}$, and $W_{111}-W_{101}$ planes for various Rayleigh numbers (increasing downward) for fixed value of $T$ and $q$.

As Rayleigh number is increased slowly, the solution changes from one wavy solution to another through a thin regime showing period doubling solutions (see the middle row of Fig. 2). The first wavy solution $S W 1$ has $2 \mathrm{D}$ mode $W_{101}$ with non-zero mean as in the absence of rotation in zero $\mathrm{P}$ convection [12, while the second wavy solution $S W 2$ has 2D mode with zero mean. As Rayleigh number is increased, the exchange of energy from 2D modes to waves increases. The larger amplitude variation of vorticity modes is at the cost of energy of $2 \mathrm{D}$ rolls. This is well known feature in the case of oscillatory instability.

As $T$ is raised further, the rotation facilitates easily the exchange of more energy from the $2 \mathrm{D}$ roll mode $W_{1010}$ to the vertical vorticity mode $\zeta_{101}$ through linear coupling. We observe an interesting behavior for $T>6$ (see Fig. 1). The conduction state becomes unstable via stationary instability [13 but the final state just above onset is quasiperiodic waves [17]. Figure 3 shows the variation of various modes with time. The amplitudes of all the modes begin modulating at the same frequency. However, the ratio of the frequency of wavy motion and that of amplitude modulation not an integer. The Fourier transform of these modes shows two independent frequencies. The frequency of amplitude modulation is much smaller compared to that for wavy motion. The sharp decrease of the amplitudes of higher order modes confirms the fast convergence of the expansion. The model, therefore, represents accurately the scenario close to the instability onset. Figure 4 shows the projections of phase space trajectories in various planes. It clearly describes the quasiperiodicity of the convective flow. The trajectories are confined in twelve dimensional torous in the phase space. The quasiperiodic behavior originates due to the nonlinear interaction among the $2 \mathrm{D}$ velocity mode $W_{101}$, the $2 \mathrm{D}$ vorticity mode $\zeta_{101}$ excited by rotation, and the wavy vorticity mode $\zeta_{010}$. Figure 5 reveals some interesting details of the time dependence of of convective patterns. The complex textures of the quasiperiodic patterns are shown over a period of wavy motion, which is much faster than the amplitude modulation. The halves of a period of wavy motion are quite asymmetric. The textures of the pattern at different times are never the same due to quasiperiodicity. 
We have presented in this work a simple dynamical system, which describes the phenomenon of thermal convection in rotating Boussinesq fluid of zero Prandtl number very close to the onset. For Taylor number above a critical value $T_{c}$, quasiperiodic waves are observed at the instability onset. For very values of Taylor number below $T_{c}$, the coriolis force causes one wavy instability to another through period doubling instability. We have shown that convection might be possible as quasiperiodic waves, even if the principle of exchange of stability is valid according to linearized hydrodynamical system. The saturation to quasi-periodic convectie state is self-tuned purely due to the nonlinear effects. This is an example of new self-tuned bifurcation scenario from the conduction state to unsteady convective state. The model presented would also be useful to study an interesting possibility of transition from a state of rest to quasi-periodic chaos [18] at the primary instability.

Acknowledgements: This work was sponsored by DST, Govt. of India under its project "Pattern-forming instability and interface waves". 


\section{References}

[1] E. A. Spiegel, J. Geophys. Res. 67, 3062 (1962).

[2] R. H. Kraichnan and E. A. Spiegel, Phys. Fluids 5, 583 (1962).

[3] J. R. Herring, Woods Hole Oceanogr. Inst. Tech. Rep. WHOI-70-01 (1970).

[4] M. R. Proctor, J. Fluid Mech. 82, 97 (1977).

[5] P. L. Sulem, C. Sulem, and O. Thual, Prog. Astro. Aeronaut. 100, 125 (1985).

[6] A. Chiffaudel, S. Fuave, and B. Perrin, Europhys. Lett. 4, 555 (1987).

[7] V. Croquette, Contemp. Phys. 30, 113 (1989); 30, 153 (1989).

[8] K. Kumar, Woods Hole Oceanogr. Inst. Tech. Rep. WHOI-90-01 (1990).

[9] R. M. Clever and F. H. Busse, Phys. Fluids A2, 334 (1990).

[10] P. Pal and K. Kumar, nlin.PS/0103056 (2001).

[11] O. Thual, J. Fluid Mech. 240, 229 (1992).

[12] K. Kumar, S. Fauve and O. Thual, J. Phys. II, France 6, 945 (1996).

[13] S. Chandrasekhar, Hydrodynamic and Hydromagnetic Stability (Oxford University Press, New York, 1961).

[14] J. B. McLaughlin and P. C. Martin, Phys. Rev. A 12, 186 (1975).

[15] S. Chaudhuri, M. Stat. Dissertation, Indian Statistical Institute, Calcutta (2000); S. Chaudhuri, A. Das, and K Kumar (2001) (to be published).

[16] see for effects of rotation on symmetries of convective patterns, G. Veronis, J. Fluid Mech. 5, 401 (1957).

[17] e.g., M. Dubois and P. Bergé, J. Phys. Letts., France 42, 167 (1981).

[18] D. Ruelle and F. Takens, Comm. Math. Phys. 20, 167 (1971); 23344 (1971). 


\section{Figure Caption}

Fig. 1: Stability boundaries in the parameter space $R-T$ just above the onset of convection. The conduction state is stable below the lowest straight line, which shows critical value $R_{c}$ of Rayleigh number as a function of Taylor number $T\left(q / K_{c}=0.4\right)$. The region marked $Q P$ shows quasiperiodic waves. The regions denoted by $S W 1$ and $S W 2$ show two regimes of wavy solutions separated by a thin region where period-doubling is observed.

Fig. 2: Phase portrait for various values of Rayleigh number $R$ ( $T=2.0$ and $\left.q / k_{c}=0.4\right)$. The top row, starting from left, shows the plots of modes $\zeta_{101}, \zeta_{010}$, and $W_{111}$ with respect to $W_{101}$ for $R=663.0$. The middle and the bottom rows show the same plots for $R=664.95$ and $R=668$ respectively. The top and the bottom rows two different wavy regimes $S W 1$ and $S W 2$ respectively, while the middle row represents period doubling corresponding to the narrow regime between $S W 1$ and $S W 2$ in Figure 1.

Fig. 3: Time variation of all the modes for $T=10.0, q / k_{c}=0.4$, and $R=678.0$ long after all transients are died out . The critical Rayleigh number $R_{c}(T=10)=677.0768$. Starting from left, the top row shows variation of $W_{101}, W_{\overline{1} 11}, W_{111}$, and $W_{012}$ with time. The middle row shows variation of $\zeta_{101}, \zeta_{010}, \zeta_{111}, \zeta_{200}$, and the bottom row shows variation of $\zeta_{012}, \zeta_{210}, \zeta_{\overline{2} 10}$, and $\zeta_{\overline{1} \overline{1} 0}$.

Fig. 4: Phase space portraits showing quasiperiodic motion for $T=10.0$, $q / k_{c}=0.4$, and $R=677.08$. Starting clockwise from the left top, they show the projections of of the phase space in $W_{101}-W_{\overline{1} \overline{1} 1}, \zeta_{101}-W_{101}, \zeta_{\overline{2} \overline{1} 0}-W_{101}$, and $W_{012}-W_{101}$ planes. Some modes like $\zeta_{\overline{2} \overline{1} 0}$ and $W_{012}$ have faster time period (i.e., period of wavy oscillation) double that of $W_{101}$.

Fig. 5: Contour plots $\left(T=7.0, q / k_{c}=0.4, R=671.30\right)$ at $z=0.25$. Various textures of the pattern is shown over a period of faster time scale

$t_{0}$ at the equal time interval of $t_{0} / 8$. The sequence of time evolution of the pattern-texture is shown from left to right in each row starting from the top row. 
This figure "fig1.jpg" is available in "jpg" format from: http://arxiv.org/ps/physics/0104036v3 
This figure "fig2.jpg" is available in "jpg" format from: http://arxiv.org/ps/physics/0104036v3 
This figure "fig3.jpg" is available in "jpg" format from: http://arxiv.org/ps/physics/0104036v3 
This figure "fig4.jpg" is available in "jpg" format from: http://arxiv.org/ps/physics/0104036v3 
This figure "fig5.jpg" is available in "jpg" format from: http://arxiv.org/ps/physics/0104036v3 